\title{
Diminuição dos casos notificados de coqueluche em crianças brasileiras: reflexo do distanciamento social e suspensão das aulas devido pandemia de Covid-19
}

Decrease in reported cases of pertussis in Brazilian children: a reflection of social distancing and suspension of classes due to the Covid-19 pandemic

Disminución de casos reportados de tos ferina en niños brasileños: reflejo del distanciamiento social y suspensión de clases por la pandemia Covid-19

Bárbara Queiroz de Figueiredo ORCID: https://orcid.org/0000-0003-1630-4597 Centro Universitário de Patos de Minas, Brasil

E-mail: barbarafigueiredo@unipam.edu.br

Andressa Ferreira Andrade

ORCID: https://orcid.org/0000-0002-9546-6596 Centro Universitário de Patos de Minas, Brasil E-mail: andressafa@unipam.edu.br

Iuri Pimenta Oliveira

ORCID: https://orcid.org/0000-0002-9004-1513 Centro Universitário de Patos de Minas, Brasil

E-mail: iuripimenta@unipam.edu.br

João Pedro Gomes de Oliveira

ORCID: https://orcid.org/0000-0002-1215-0423 Centro Universitário de Patos de Minas, Brasil E-mail: joaogomes@unipam.edu.br

Karine Soares Ferreira

ORCID: https://orcid.org/0000-0002-6110-2671

Centro Universitário de Patos de Minas, Brasil E-mail: f.karinesoares@gmail.com

Leomar dos Santos Silva

ORCID: https://orcid.org/0000-0003-4386-5656

Centro Universitário de Patos de Minas, Brasil E-mail: leomarss@unipam.edu.br

Maria Caroline Takahashi dos Santos ORCID: https://orcid.org/0000-0002-2234-4440

Centro Universitário de Patos de Minas, Brasil E-mail:mariactakahashis@gmail.com

Mauro Soares Marra

ORCID: https://orcid.org/0000-0002-0543-1569

Centro Universitário Atenas, Brasil

E-mail: maurosoaresm@gmail.com

Stéfani do Vale

ORCID: https://orcid.org/0000-0002-5211-9834

Centro Universitário Atenas, Brasil

E-mail: stefani.vale@hotmail.com

\begin{abstract}
Resumo
Introdução: a coqueluche é uma doença infecciosa aguda de alta transmissibilidade e uma importante causa de morbimortalidade infantil. É causada pelas bactérias Bordetella pertussis e B. parapertussis, sendo o homem seu único reservatório natural, acometendo o aparelho respiratório, principalmente a traqueia e os brônquios. É transmitida, principalmente, pelo contato direto da pessoa doente com uma pessoa suscetível, não vacinada, através de gotículas de saliva expelidas por tosse, espirro ou saliva, bem como pelo contato com objetos contaminados com secreções do doente. Objetivo: atestar, por meio de dados públicos de notificações de casos de incidência desta doença, a queda desses números no Brasil. em 2020, em comparação ao ano anterior. Metodologia: Trata-se de um estudo epidemiológico descritivo e quantitativo. Foi realizada a busca por casos de coqueluche nos anos de 2019 e 2020, registrados no Sistema de Informação de Agravos de Notificação (SINAN) - DATASUS. Resultados $e$ discussão: o fechamento de escolas e creches levou a uma redução imediata e dramática dos contatos entre as crianças e, consequentemente, das infecções transmissíveis, que são a causa de grande parte das consultas de saúde pediátrica Conclusão: a redução dramática das doenças infecciosas transmissíveis leva a uma oportunidade de estudar a
\end{abstract}


incidência de diagnósticos que são presumidos, mas não provados definitivamente como sendo causados por infecções. Embora a base de evidências circunstanciais para esse efeito seja ampla, a análise atual adiciona ainda mais a isso, haja vista que redução relativa nos diagnósticos presumidos de infecção pode ser tão grande quanto a redução nas infecções transmissíveis.

Palavras-chave: Coqueluche; Infecção; Crianças; Bordetella pertussis; Epidemiologia.

\begin{abstract}
Introduction: Pertussis is an acute infectious disease of high transmissibility and an important cause of childhood morbidity and mortality. It is caused by the bacteria Bordetella pertussis and B. parapertussis, and man is its only natural reservoir, affecting the respiratory system, especially the trachea and bronchi. It is mainly transmitted by the direct contact of the sick person with a susceptible, unvaccinated person, through droplets of saliva expelled by coughing, sneezing or saliva, as well as by contact with objects contaminated with the patient's secretions. Objective: to attest, through public data of notifications of cases of incidence of this disease, the drop in these numbers in Brazil. in 2020, compared to the previous year. Methodology: This is a descriptive and quantitative epidemiological study. The search for pertussis cases in the years 2019 and 2020, registered in the Information System for Notifiable Diseases (SINAN) - DATASUS, was carried out. Results and discussion: the closing of schools and day care centers led to an immediate and dramatic reduction in contacts between children and, consequently, in communicable infections, which are the cause of most pediatric health consultations Conclusion: the dramatic reduction in infectious diseases Communicable diseases leads to an opportunity to study the incidence of diagnoses that are presumed but not definitively proven to be caused by infections. Although the circumstantial evidence base for this effect is broad, the current analysis adds even more to this, as the relative reduction in presumed diagnoses of infection can be as large as the reduction in communicable infections.
\end{abstract}

Keywords: Whooping cough; Infection; Children; Bordetella pertussis; Epidemiology.

\title{
Resumen
}

Introducción: una coqueluche é uma doença infecciosa aguda de alta transmissibilidade e uma importante causa de morbimortalidade infantil. É causada pelas bactérias Bordetella pertussis e B. parapertussis, sendo o homem seu único reservatório natural, acometendo o aparelho respiratório, principalmente a traqueia e os brônquios. É transmitida, principalmente, pelo contato direto da pessoa doente com uma pessoa suscetível, não vacinada, através de gotículas de saliva expelidas por tosse, espirro ou saliva, bem como pelo contato com objetos contaminados com secreções doente. Objetivo: atestar, por meio de dados públicos de notificações de casos de incidência desta doença, a queda desses números no Brasil. em 2020, em comparação ao ano anterior. Metodología: Trata-se de um estudo epidemiológico descritivo e quantitativo. Foi realizada a busca por casos de coqueluche nos anos de 2019 y 2020, registrados no Sistema de Informação de Agravos de Notificação (SINAN) - DATASUS. Resultados e Discusion: o fechamento de escolas e creches levou a uma redução imediata e dramática dos contatos entre as crianças e, Consecuentemente, das infecções transmissíveis, que são a causa de grande parte das consultas de saúde pediátrica Conclusão: a redução dramática das doenças infecciosas transmissíveis leva a una oportunidad de estudar a incidência de diagnósticos que são presumidos, mas no provados definitivamente como sendo causados por infecções. Embora a base de evidências circunstanciais para esse efeito seja ampla, a análise atual adiciona ainda mais a isso, haja vista que redução relativa nos diagnósticos presumidos de infecção pode ser tão grande quanto a redução nas infecções transmissíveis.

Palabras clave: Coqueluche; Infecção; Crianças; Bordetella pertussis; Epidemiologia.

\section{Introdução}

A coqueluche é uma doença infecciosa aguda de alta transmissibilidade e uma importante causa de morbimortalidade infantil. É causada pelas bactérias Bordetella pertussis e B. parapertussis, sendo o homem seu único reservatório natural, acometendo o aparelho respiratório, principalmente a traqueia e os brônquios (Medeiros et al. 2017). É transmitida, principalmente, pelo contato direto da pessoa doente com uma pessoa suscetível, não vacinada, através de gotículas de saliva expelidas por tosse, espirro ou saliva, bem como pelo contato com objetos contaminados com secreções do doente. A coqueluche é especialmente transmissível na fase catarral e em locais com aglomeração de pessoas. Geralmente, dentre os sintomas, destaca-se a febre inicial, dores pelo corpo, mal-estar, sinais de congestão e tosse forte e constante, que pode se prolongar por semanas, mesmo depois que os outros sintomas respiratórios diminuíram de intensidade, podendo até mesmo corroborar apneia (Belini et al. 2021).

A doença é imunoprevenível e existem dois tipos de vacina contra coqueluche: a vacina baseada em organismos de $B$. pertussis mortos (vacina de célula inteira); e a vacina acelular, baseada em componentes altamente purificados da $B$. 
pertussis. Ambas as vacinas apresentam excelentes registros de segurança, no entanto, devido sua reatogenicidade, a vacina composta por células inteiras é recomendada para crianças menores de sete anos de idade. A imunidade conferida pela vacina é duradoura, mas não permanente, decrescendo com o tempo, até que sua proteção chegue a se mostrar bastante reduzida, ou nenhuma: o que acontece, em média, no prazo de 5 a 10 anos após a última dose administrada (Willemann et al. 2014). No Brasil, a coqueluche é uma doença de notificação compulsória. Os dados relativos à notificação e investigação dessa doença são armazenados no Sistema de Informação de Agravos de Notificação (SINAN).

Sob contexto de pandemia por infecção por SARS-CoV-2, em meio às enormes dificuldades enfrentadas pelas crianças na quarentena (desde o isolamento até a interrupção nas aulas presenciais), houve queda na incidência de doenças infecciosas infantis simples, como resfriados, ou graves, como bronquiolite, que lotam hospitais pediátricos, bem como de coqueluche (Angoulvant et al. 2020). Sob esse contexto, o presente estudo tem como objetivo atestar, por meio de dados públicos de notificações de casos de incidência desta doença, a queda desses números no Brasil em 2020, em comparação ao ano anterior.

\section{Metodologia}

Trata-se de um estudo epidemiológico descritivo e quantitativo. Foi realizada a busca por casos de coqueluche nos anos de 2019 e 2020, registrados no Sistema de Informação de Agravos de Notificação (SINAN) - DATASUS, com base no recorte espacial das cinco regiões brasileiras (Norte, Nordeste, Sudeste, Sul e Centro-Oeste), das faixas etárias, dos critérios diagnósticos e dos meses referentes aos primeiros sintomas. O presente trabalho foi realizado nas seguintes etapas: (I) pesquisa bibliográfica, (II) análise e coleta dos casos de coqueluche no Brasil, nos anos de 2019 e 2020, (III) coleta destes dados, também por regiões, (IV) quantificação dos casos de acordo com faixa etária, (V) análise dos casos de acordo com os meses do ano de 2020.

O presente estudo contou com o aporte de trabalhos acadêmicos entre os anos de 2020 e 2021. Por meio do acesso às principais bases de dados, a saber: dados National Library of Medicine (PubMed MEDLINE), Scientific Electronic Library Online (Scielo), Cochrane Database of Systematic Reviews (CDSR), Google Scholar, Biblioteca Virtual em Saúde (BVS) e EBSCO Information Services, no mês de agosto 2021. Para a compreensão visual dos resultados, utilizou-se dos dados disponibilizados pelo DATASUS para a construção de gráficos tabelas. Por fim, a análise desses dados foi crucial para verificação de que a incidência dos casos de coqueluche, principalmente em crianças, obteve queda significativa no ano de 2020, quando comparada ao ano de 2019.

\section{Resultados e Discussão}

Identificou-se a ocorrência dos casos notificados de coqueluche nos anos de 2019 e 2020, no Brasil, a fim de estabelecer uma relação comparativa, conforme ilustrada na Tabela 1 e no Gráfico 1.

Tabela 1: Casos confirmados, segundo ano de primeiro sintoma, por faixa etária, nos anos de 2019 e 2020

\begin{tabular}{c|c|c|c|c|c|c|c|c|c|c|c}
\hline $\begin{array}{c}\text { ANO DE } \\
\text { PRIMEIRO } \\
\text { SINTOMA }\end{array}$ & $\begin{array}{c}<\mathbf{1} \\
\text { ANO }\end{array}$ & $\begin{array}{c}\mathbf{1 - 4} \\
\text { ANOS }\end{array}$ & $\begin{array}{c}\mathbf{5 - 9} \\
\text { ANOS }\end{array}$ & $\begin{array}{c}\mathbf{1 0 - 1 4} \\
\text { ANOS }\end{array}$ & $\begin{array}{c}\mathbf{1 5 - 1 9} \\
\text { ANOS }\end{array}$ & $\begin{array}{c}\mathbf{2 0 - 3 9} \\
\text { ANOS }\end{array}$ & $\begin{array}{c}\mathbf{4 0 - 5 9} \\
\text { ANOS }\end{array}$ & $\begin{array}{c}\mathbf{6 0 - 6 4} \\
\text { ANOS }\end{array}$ & $\begin{array}{c}\mathbf{6 5 - 6 9} \\
\text { ANOS }\end{array}$ & $\begin{array}{c}\mathbf{8 0 ~ E +} \\
\text { ANOS }\end{array}$ & TOTAL \\
\hline 2019 & 799 & 315 & 101 & 125 & 25 & 97 & 46 & 6 & 2 & 3 & 1.519 \\
\hline 2020 & 126 & 41 & 8 & 9 & 3 & 19 & 10 & 1 & 1 & 0 & 218 \\
\hline
\end{tabular}

Fonte: TABNET - DATASUS (2021). 
Gráfico 1: Casos confirmados, segundo ano de primeiro sintoma, por faixa etária, nos anos de 2019 e 2020.

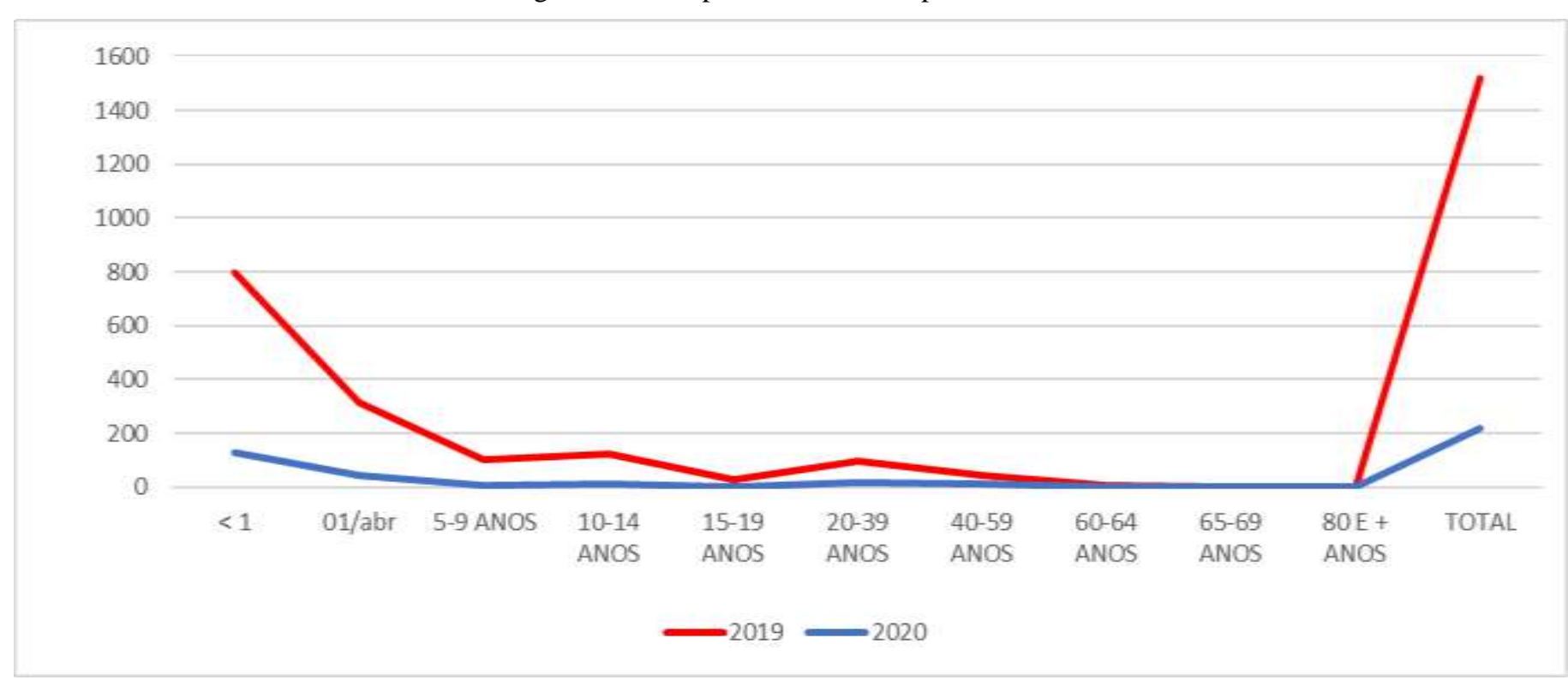

Fonte: TABNET - DATASUS (2021).

Nota-se, portanto, que em relação a 2019, os casos de coqueluche, no Brasil, tiveram diminuição exponencial, principalmente em crianças de até 14 anos. Sob essa perspectiva, elaborou-se uma tabela (Tabela 2), quantificando os casos confirmados de coqueluche segundo mês do primeiro sintoma, em 2020. Além disso, a Tabela 3 é capaz de ilustrar as regiões do Brasil quantificadas aos números de casos confirmados de coqueluche, também em 2020.

Tabela 2: Casos confirmados, segundo mês de primeiro sintoma, no ano de 2020.

\begin{tabular}{c|c}
\hline MES DE PRIMEIRO SINTOMA & CASOS CONFIRMADOS \\
\hline JANEIRO & 82 \\
\hline FEVEREIRO & 50 \\
\hline MARÇO & 23 \\
\hline ABRIL & 15 \\
\hline MAIO & 10 \\
\hline JUNHO & 8 \\
\hline JULHO & 7 \\
\hline AGOSTO & 6 \\
\hline SETEMBRO & 6 \\
\hline OUTUBRO & 6 \\
\hline NOVEMBRO & 3 \\
\hline DEZEMBRO & 2 \\
\hline TOTAL & 218 \\
\hline
\end{tabular}

Fonte: TABNET - DATASUS (2021).

Tabela 3: Casos confirmados, segundo região de notificação, no ano de 2020.

\begin{tabular}{c|c}
\hline REGIÃO DE NOTIFICAÇÃO & CASOS CONFIRMADOS \\
\hline SUDESTE & 80 \\
\hline NORDESTE & 68 \\
\hline SUL & 44 \\
\hline NORTE & 15 \\
\hline CENTRO-OESTE & 11 \\
\hline TOTAL & 218 \\
\hline
\end{tabular}

Fonte: TABNET - DATASUS (2021). 
Ademais, a Tabela 4 expõe os critérios diagnósticos utilizados para confirmação de casos de coqueluche, em 2020, ressaltando, por meio quantitativo, que os critérios clínicos foram os mais utilizados.

Tabela 4: Casos confirmados, segundo critérios diagnósticos e confirmatórios, no ano de 2020.

\begin{tabular}{c|c}
\hline CRITÉRIO CONFIRMATÓRIO & CASOS CONFIRMADOS \\
\hline CLÍNICO & 105 \\
\hline LABORATÓRIO & 65 \\
\hline CLÍNICO-EPIDEMIOLÓGICO & 45 \\
\hline IGN/BRANCO & 3 \\
\hline TOTAL & 218 \\
\hline
\end{tabular}

Fonte: TABNET - DATASUS (2021).

A Tabela 5 foi elaborada a fim de elucidar os desfechos dos pacientes infantis, dentre 0 e 4 anos, acometidos por coqueluche, em 2020, em que a maioria evoluiu com cura da doença.

Tabela 5: Casos de cura e óbitos por coqueluche em crianças, no ano de 2020.

\begin{tabular}{c|c|c}
\hline FAIXA ETÁRIA & CURA & ÓBITOS PELO AGRAVO NOTIFICADO \\
\hline$<1$ ANO & 114 & 1 \\
\hline $1-4$ & 34 & 1 \\
\hline TOTAL & 148 & 2 \\
\hline
\end{tabular}

Fonte: TABNET - DATASUS (2021).

Sob a perspectiva encontrada pelos resultados deste estudo, nota-se que o fechamento de escolas e creches levou a uma redução imediata e dramática dos contatos entre as crianças e, consequentemente, das infecções transmissíveis, que são a causa de grande parte das consultas de saúde pediátrica (Kruizinga et al. 2021). O estudo atual apoia essa hipótese, visto que a maior redução observada nos casos notificados de coqueluche no Brasil, quando comparados ao ano de 2019. Aliado a isso, um segundo possível fator de influência pode ter sido o fato de que a pandemia levou a uma demanda extrema no setor de saúde e que o atendimento clínico no hospital girou em grande parte em torno de pacientes com Covid-19 (Kubicek et al. 2020).

O medo da infecção por SARS-CoV-2 e o desejo de não aumentar ainda mais a demanda no setor de saúde podem ter feito os pais se absterem de ir ao hospital com seus filhos, apesar do agravamento dos sintomas. Além disso, ainda é possível que os pais e outros cuidadores sofram menos pressão do que o normal para procurar atendimento clínico imediato para seus filhos em caso de doença (Goldman et al. 2020). Ou seja, não está claro se essa redução se deve apenas a uma diminuição nas infecções transmissíveis ou também por mudanças comportamentais em torno da utilização de cuidados de saúde, haja vista exemplos relatados de evitação de cuidados devido ao medo de um ambiente hospitalar, o que seria potencialmente desastroso.

\section{Conclusão}

A redução dramática das doenças infecciosas transmissíveis leva a uma oportunidade de estudar a incidência de diagnósticos que são presumidos, mas não provados definitivamente como sendo causados por infecções. Embora a base de evidências circunstanciais para esse efeito seja ampla, a análise atual adiciona ainda mais a isso, haja vista que redução relativa nos diagnósticos presumidos de infecção pode ser tão grande quanto a redução nas infecções transmissíveis (Alfano et al. 2020). Além disso, o isolamento social como intervenção permite uma oportunidade única de investigar a incidência de diagnósticos que se presume, mas que ainda não foi definitivamente comprovado, como relacionados ou associados a infecções 
Research, Society and Development, v. 10, n. 11, e230101119631, 2021

(CC BY 4.0) | ISSN 2525-3409 | DOI: http://dx.doi.org/10.33448/rsd-v10i11.19631

transmissíveis, exemplo da coqueluche, evidenciada neste estudo. No entanto, é de suma importância que estudos posteriores atestem esta relação, haja vista as limitações apresentadas neste estudo.

\section{Referências}

Alfano, V., \& Ercolano S. (2020). A eficácia do bloqueio contra COVID-19: uma análise de painel cross-country. Appl Health Econ Health Policy. 18 (4): $509-517$.

Angoulvant, F., Ouldali, N., Yang, D. D., Filser, M., Rybak, A., Gajdos, V., Beaujouan, L., \& Skurnik, D. (2020). Pandemia de COVID-19: impacto causado pelo fechamento de escolas e bloqueio nacional em consultas pediátricas e admissões por infecções virais e não virais, uma análise de série temporal. Clin Infect Dis., 72 (2), 319-322.

Bellini, G. F., Sartori, G. P., Pellizzaro, A. O., Santos, E. L., \& Batista, K. Z. S. (2021). Incidência de infecções respiratórias em crianças até 4 anos: relação com o cumprimento do calendário vacinal. Revista Ciência e Saúde, 6 (1), 18-23.

Goldman, R. D., Grafstein, E., Barclay, N., Irvine, M. A., \& Portales-Casamar, E. (2020). Pacientes pediátricos vistos em 18 departamentos de emergência durante a pandemia de COVID-19. Emerg Med J. 37 (12): 773-777.

Kruizinga, M. D., Peeters, D., van Veen, M., van Houten, M., Wieringa, J., Noordzi, J. G., Bekhof, J., Stranders, G. T., Vet, N. J., \& Driessen, G. J. A. (2021). O impacto do bloqueio nas visitas pediátricas ao pronto-socorro e nas internações hospitalares durante a pandemia de COVID-19: uma análise multicêntrica e revisão da literatura. Eur J Pediatr., 180 (7), 2271-2279.

Kubicek, K., Liu, D., Beaudin, C., Supan, J., Weiss, G., Lu, Y., \& Kipke, M. D. (2021). Um perfil do uso de serviços de emergência não urgentes em um hospital pediátrico urbano. Pediatr Emerg Care. 28 (10): 977-984.

Medeiros, A. T. N., Cavalcante, C. A., Souza, N. L., \& Ferreira, M. A. F. (2017). Reemergência da coqueluche: perfil epidemiológico dos casos confirmados. Cadernos de Saúde Coletiva, 25 (4), 453-459.

Ministério da Saúde. Departamento de informática do SUS. (2021). Brasília, DF. <http://datasus.saude.gov.br/informacoes-de-saude>.

Willemann, M. C. A., Goes, F. C. S., Araújo, A. C. M., \& Domingues, C. M. A. S. (2014). doecimento por coqueluche e número de doses administradas de vacinas Pertussis. Epidemiologia e Serviços de Saúde, 23 (2), 1-10. 